Nachwuchs früh gewinnen

\title{
Vorschulkinder auf Erkundungstour im St. Hedwig Krankenhaus
}

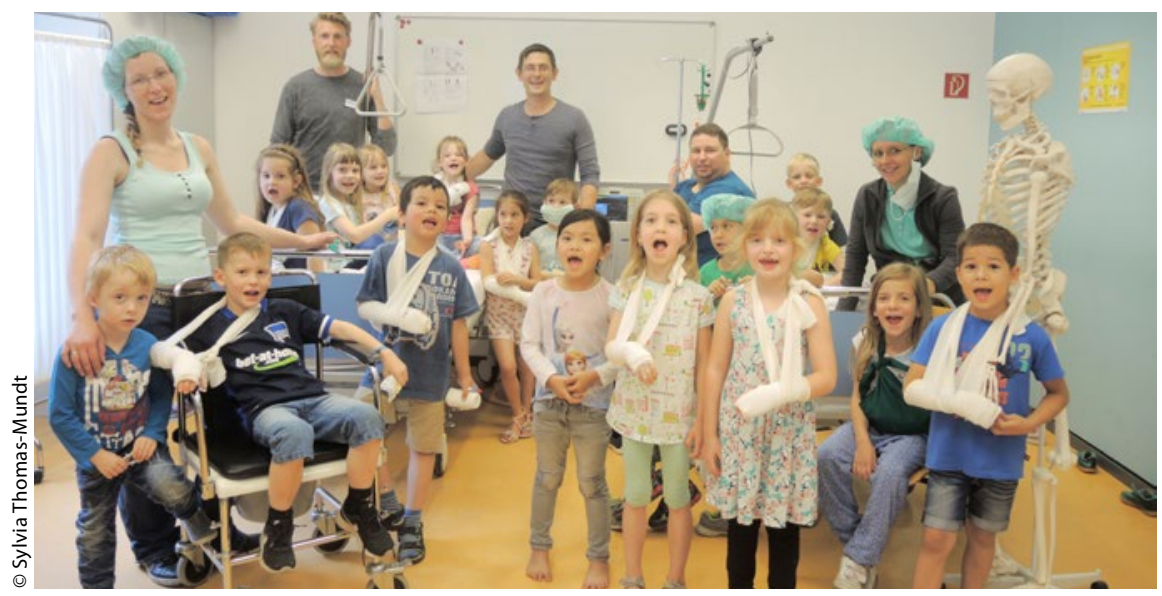

Vorschulkinder aus der Berliner KiTA „Sophies Welt" auf Erkundung im Berliner St. Hedwig Krankenhaus. Unser Autor, selbst Krankenpfleger, hinten in der Bildmitte.

- Junge Menschen an den Pflegeberuf heranzuführen, gewinnt immer mehr an Bedeutung. Aber wo fängt man an? Am besten an dem Punkt, an dem Orientierung beginnt - und zwar im frühen Kindesalter. Eine Gruppe von Vorschulkindern aus der KiTa "Sophies Welt" wollte Arbeitsplätze der Eltern genauer anschauen. Nach den Klassi-

\section{Augusta-Viktoria-Stift geht online Whatsapp \& Co.}

\section{— Pflegebedürftige Senioren sind von der Kommunikation über moderne Me- dien meist ausgeschlossen. Die Mitarbei- ter des Augusta-Viktoria-Stifts in Erfurt wollten sich damit nicht zufrieden geben und den Bewohnern die Nutzung von Whatsapp und Skype ermöglichen. Dazu soll ein Internetzugang zur freien Ver- fügbarkeit eingerichtet werden. Mit pri- vaten Geräten kann so der Kontakt zu Familie und Bekannten gehalten wer- den. Der soziale Dienst des Hauses wird zusätzlich mit Tablets ausgestattet. Er- möglicht wurden die Investitionen durch die Share Value Stiftung.}

www.augusta-viktoria-stift.de kern - Feuerwehr, Polizei und Luftfahrt stand das Gesundheitswesen im Fokus.

Wie konnte man die Wünsche der Kinder aber mit einem Krankenhaus verbinden? Könnte man Kinder auf eine Station mitnehmen, würden sie dort den Ablauf stören oder Dinge sehen, die sie überfordern würden? Wären die Eltern damit einverstan- den? Die Hindernisse schienen groß, wurden aber schnell überwunden. Die Krankenpflegeschule des St. Hedwig Krankenhauses in Berlin-Mitte erwies sich als geeignet, durch einen Lehrer wurden der Übungsraum für den Besuch reserviert und das Kollegium informiert.

Bei Ankunft der Kinder auf dem Gelände und dem Weg zur Schule wichen die zunächst noch skeptischen Blicke beim Betreten des Raumes und der Sicht auf das Skelett einem Staunen. Nach kurzer Beschreibung einiger Tätigkeiten in einer Klinik konnten die Kinder nun erfahren, was sie in einem Krankenhaus erwarten würde. Gezeigt und erklärt wurden das Verbinden, ein EKG-Gerät sowie fahrbare Betten. Highlight: das Anlegen eines Gipses durch einen Mitarbeiter aus der Notaufnahme. Dadurch konnten die Kinder selbst fühlen, wie es ist, wenn die Handlungsfähigkeit eingeschränkt ist. Faszinierend war es zu beobachten, wie die Mädchen und Jungen damit begannen, sich untereinander zu helfen. Natürlich wirkten auch die beiden begleitenden Erzieherinnen aktiv mit.

Viele Aufgaben der Pflegenden waren den Kindern nicht bekannt. Wenn es darum geht, den Pflegeberuf attraktiver zu gestalten und das Image der Pflege aufzubauen, war dieser Tag ein Erfolg. Schade nur, dass die Bewerbungsfrist erst in zehn Jahren abläuft.

(Markus Meißner)

\section{Kunstaktion in der Kinderonkologie}

\section{Schaltung zur Internationalen Raumstation}

— Auf der Station der Kinderonkologie der Uniklinik Köln gab es Ende August eine LiveSchalte auf die Internationale Raumstation (ISS). Im Herbst 2016 waren die beiden ehemaligen NASA-Astronauten Ian Cion und Nicole Stott auf Station zu Gast. Sie bemalten gemeinsam mit den krebskranken Kindern kleine Stoffstücke. Später wurden diese zu einem Raumanzug zusammengenäht. Jetzt trug ein Astronaut diesen besonderen Raumanzug auf der ISS, beantwortete während der Schalte Fragen der krebskranken Kinder und schwebte mit dem Anzug in der Schwerelosigkeit.

Das Projekt wird von dem führenden Krebscenter der University of Texas (MD Anderson Cancer Center) in Zusammenarbeit mit der NASA und ESA initiiert. An der Aktion nahmen bislang über 600 Kinder weltweit teil.

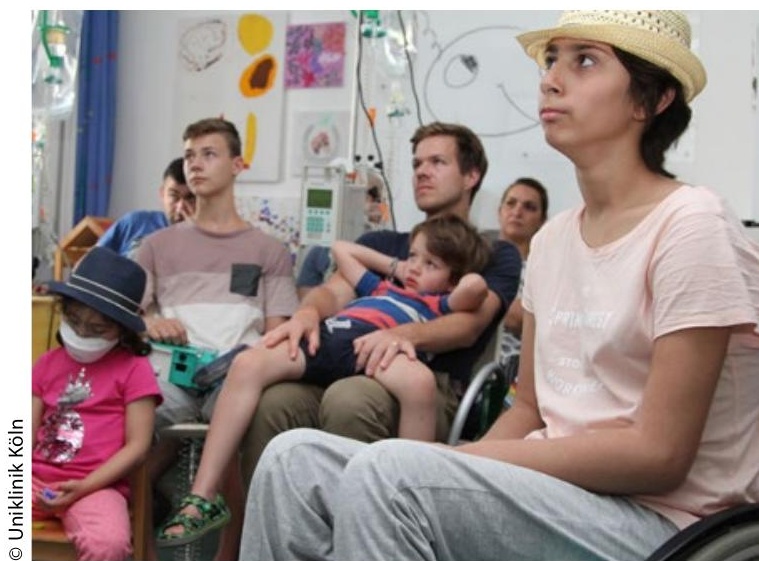

Live-Schaltung aus dem Weltall in die Kinderonkologie

www.uk-koeln.de 